\title{
An Inequality for Doubly Stochastic Matrices*
}

\author{
Charles R. Johnson** and R. Bruce Kellogg** \\ Institute for Basic Standards, National Bureau of Standards, Washington, D.C. 20234
}

(June 30, 1976)

\begin{abstract}
Interrelated inequalities involving doubly stochastic matrices are presented. For example, if $B$ is an $n$ by $n$ doubly stochastic matrix, $x$ any nonnegative vector and $y=B x$, then $x_{1} x_{2} \cdots x_{n} \leq$ $y_{1} y_{2} \cdots y_{n}$. Also, if $A$ is an $n$ by $n$ nonnegative matrix and $D$ and $E$ are positive diagonal matrices such that $B=D A E$ is doubly stochastic, then $\operatorname{det} D E \geq \rho(A)^{-n}$, where $\rho(A)$ is the PerronFrobenius eigenvalue of $A$. The relationship between these two inequalities is exhibited.
\end{abstract}

Key words: Diagonal scaling; doubly stochastic matrix; Perron-Frobenius eigenvalue.

An $n$ by $n$ entry-wise nonnegative matrix $B=\left(b_{i j}\right)$ is called row (column) stochastic if $\sum_{j=1}^{n} b_{i j}=1$ for all $i=1, \cdots, n\left(\sum_{i=1}^{n} b_{i j}=1\right.$ for all $\left.j=1, \cdots, n\right)$. If $B$ is simultaneously row and column stochastic then $B$ is said to be doubly stochastic. We shall denote the Perron-Frobenius (maximal) eigenvalue of an arbitrary $n$ by $n$ entry-wise nonnegative matrix $A$ by $\rho(A)$. Of course, if $A$ is stochastic, $\rho(A)=1$.

It is known precisely which $n$ by $n$ nonnegative matrices may be diagonally scaled by positive diagonal matrices $D, E$ so that

$$
B=D A E
$$

is doubly stochastic. If there is such a pair $D, E$, we shall say that $A$ has property (*). In this event it is our interest to obtain inequalities on $D$ and $E$. In the process, certain related inequalities for doubly stochastic matrices are noticed.

It was first realized by Sinkhorn [4] ${ }^{1}$ that if $A$ is entry-wise positive and square, then $A$ has property $(*)$. The proof amounts to showing that the process of alternately scaling $A$ to produce a row stochastic matrix, and then a column stochastic matrix, and then continuing the process, actually converges to a doubly stochastic matrix. The hypothesis of positivity, however, can be weakened somewhat. If there exists no single permutation matrix $P$ such that

$$
P^{T} A P=\left(\begin{array}{ll}
A_{11} & 0 \\
A_{21} & A_{22}
\end{array}\right)
$$

where $A_{11}$ and $A_{22}$ are square, then $A$ is called irreducible. If there is a pair of permutation matrices $P, Q$ such that $C=P A Q$, then we shall say that $A$ and $C$ are equivalent. If, further, $A$ is equivalent to no matrix of the form

1 Figures in brackets indicate the literature references at the end of this paper. 


$$
\left(\begin{array}{ll}
A_{11} & 0 \\
A_{21} & A_{22}
\end{array}\right)
$$

where $A_{11}$ and $A_{22}$ are square, then $A$ is termed completely irreducible. It is an easy calculation to show that $A$ has property (*) if and only if each matrix equivalent to $A$ does, and it is equally clear that for $A$ to have property $(*)$ it must have the zero-nonzero sign pattern of a doubly stochastic matrix. For example, if $A$ has property (*) and if $A$ is of form (2) this means we must then have $A_{21}=0$. It has further been shown [1] that when $A$ is completely irreducible the alternate scaling process of Sinkhorn still converges and thus $A$ has property (*). Since property (*) is preserved under direct summation, we may summarize as follows.

REMARK 1: A square nonnegative matrix A has property (") if and only if A is completely irreducible or A is equivalent to a direct sum of completely irreducible matrices.

Thus, property $\left({ }^{*}\right)$ depends only on the zero pattern of $A$. It is also a straightforward calculation (following [4] ) that

REMARK 2: If A has property (*), then the product DE of (1) is unique.

Our first observation is both necessary for later proofs and of interest by itself.

Theorem 1 : If $\mathrm{B}=\left(\mathrm{b}_{\mathrm{ij}}\right)$ is an $\mathrm{n}$ by $\mathrm{n}$ doubly stochastic matrix and $\mathrm{x} \geq 0$ is any nonnegative vector, then, for $\mathrm{y}=\mathrm{Bx}$, we have

$$
\prod_{i=1}^{n} x_{i} \leq \prod_{i=1}^{n} y_{i}
$$

If $\mathrm{B}$ is completely irreducible, equality holds in (3) if and only if the right-hand side is 0 or all components of $\mathrm{x}$ are the same. Furthermore, among all irreducible nonnegative square matrices $\mathrm{B}$ satisfying $\rho(\mathrm{B}) \leq 1$, only those diagonally similar to doubly stochastic matrices satisfy (3) for all $\mathrm{x} \geq 0$.

Proof: From the arithmetic-geometric mean inequality [2]

$$
\prod_{i=1}^{n} x_{i}^{\gamma_{i}} \underset{i=1}{\sum} \sum^{n} \gamma_{i} x_{i}
$$

where $x=\left(x_{1}, \cdots, x_{n}\right)^{T}$ is any nonnegative vector, and $\gamma=\left(\gamma_{1}, \cdots, \gamma_{n}\right)$ is a vector of nonnegative numbers satisfying $\sum_{i=1}^{\sum} \gamma_{i}=1$. Equality holds in (4) if and only if the $x_{i}$ 's corresponding to nonzero $\gamma_{i}$ 's are all equal. Now, suppose $B=\left(b_{i j}\right)$ is row stochastic and $y=B x, x \geq 0$. It follows from (4) that

$$
\prod_{j=1}^{n} x_{i j} \leq \sum_{j=1}^{n} b_{i j} x_{j}=y_{i}, \quad \text { for } i=1, \cdots, n .
$$

Taking a product over $i$ of both sides, we arrive at

$$
\prod_{j=1}^{n} x_{j} \sum_{i=1}^{n} b_{i j} \leq \prod_{i=1}^{n} y_{i} .
$$

If $B$ is doubly stochastic, $\sum_{i=1}^{n} b_{i j}=1$ for each $j=1, \cdots, n$, and it follows that (3) holds.

To analyze the case of equality, it is clear that equality holds in (3) if either $x$ is a vector of equal components or the right-hand side of (3) is 0 . On the other hand, if equality holds in (3) and the righthand side of (3) is not 0 , then equality must hold in (5) for each $i=1, \cdots, n$. This means that for each $i$, the $x_{j}$ 's corresponding to nonzero $b_{i j}$ 's are all equal. This, in turn, implies, by virtue of equality holding in (5) for all $i$, that $y=Q^{T} x$ for some permutation matrix $Q$. Since $B Q Q^{T} x=y$, we have that $B Q$ has $Q^{T} x=y$ as a Perron-Frobenius eigenvector (corresponding to $\rho(B Q)=1$ ). If $B$ is completely irreducible, then $B Q$ is irreducible, and, since $B Q$ is doubly stochastic, its Perron-Frobenius eigenspace is one-dimensional (because of the irreducibility) and is spanned by $(1,1, \cdots, 1)^{T}$. Therefore all components of $Q^{T} x$, and thus of $x$, are the same. It should be noted that even in case $B$ is completely irreducible it is possible that the right-hand side of (3) be 0 for a nonnegative nonzero vector $x$. For example, let 


$$
B=\left(\begin{array}{ccc}
1 / 3 & 1 / 3 & 1 / 3 \\
1 / 6 & 1 / 6 & 2 / 3 \\
1 / 2 & 1 / 2 & 0
\end{array}\right) \text { and } x=\left(\begin{array}{l}
0 \\
0 \\
1
\end{array}\right)
$$

Thus, the second statement of the theorem is proven. It should also be noted that the case of equality in (3) may similarly be analyzed if $B$ is equivalent to a direct sum of completely irreducibles. It is enough to assume $B$ is equal to a direct sum of completely irreducibles. Then, in addition to equality occurring in (3) when the right-hand side is 0 , equality occurs precisely when the components of $x$ are equal within each piece corresponding to a direct summand of $B$.

For the third statement, it is enough to assume that $B$ satisfies $\rho(B)=1$. It then follows from the irreducibility of $B$ that there is a positive diagonal matrix $D$ such that $D B D^{-1}$ is row stochastic $\left(D^{-1}\right.$ is obtained from the Perron-Frobenius eigenvector of $B$, which is positive). Since $B x=y$ if and only if $\left(D B D^{-1}\right) D x=D$ y, (3) holds for $D B D^{-1}$ if it holds for $B$, so that we may as well assume $B$ is row stochastic. Then, if $B$ is not doubly stochastic, some column sum is $<1$, so let $\theta=\Sigma_{i} b_{i j}<1$. Let $x_{i}=1, i \neq j$, and $x_{j}=1+\epsilon$. Then (3) becomes

$$
1+\epsilon \leq \prod_{\mathbf{i}}\left(1+b_{i j \epsilon}\right)=1+\theta \epsilon+0\left(\epsilon^{2}\right),
$$

which is impossible if $\epsilon>0$ is small enough. This completes the proof of the theorem.

It should be noted that essential portions of theorem 1 may also be demonstrated by a maximization argument.

ExAmple: The assumption of irreducibility in the third statement of theorem 1 cannot, in general, be relaxed. If $B=\left(\begin{array}{ll}1 & 0 \\ 1 & 1\end{array}\right)$ then $B$ satisfies (3) for all $x \geq 0$ and $\rho(B)=1$, but $B$ is not similar to a doubly stochastic matrix.

An alternate form of $(3)$ is

Corollary 1: If $\mathrm{B}=\left(\mathrm{b}_{\mathrm{ij}}\right)$ is an $\mathrm{n}$ by $\mathrm{n}$ doubly stochastic matrix, then for any $\mathrm{n}$ real numbers $\mathrm{t}_{1}, \cdots, \mathrm{t}_{\mathrm{n}}$, satisfying $\mathrm{t}_{\mathrm{i}} \geq-1, \mathrm{i}=1, \cdots, \mathrm{n}$, we have

$$
\prod_{i=1}^{n}\left(1+t_{i}\right) \leq \prod_{i=1}^{n}\left(1+\sum_{j=1}^{n} t_{j} b_{i j}\right) .
$$

Our primary observation concerns row stochastic matrices with property $\left(^{*}\right)$.

THeOREM 2: If $\mathrm{A}$ is a row stochastic matrix with property $\left({ }^{*}\right)$ and $\mathrm{D}$ and $\mathrm{E}$ are the positive diagonal matrices guaranteed by (1) then

$$
\operatorname{det} \mathrm{DE} \geq 1
$$

Furthermore, equality holds if and only if $\mathrm{A}$ is actually doubly stochastic.

Proof: As $B=\left(b_{i j}\right)$ runs through all $n$ by $n$ doubly stochastic matrices and $F=\operatorname{diag}\left\{f_{1}, \cdots, f_{n}\right\}$ runs through all positive diagonal matrices, then $A=D^{-1} B F$ runs through all row stochastic matrices with property (*) where $D=\operatorname{diag}\left\{{ }^{n} b_{1 j} f_{j}, \cdots, \Sigma_{\Sigma}^{n} b_{n j} f_{j}\right\}$. Thus, since $B=D A E$, where $E=F^{-1}$, it suffices to show that $\operatorname{det} D>\operatorname{det} F$. If we denote $\left(f_{1}, \cdots, f_{n}\right)^{T}$ by $f$, this is equivalent to saying that the product of the entries of $B f$ is greater than or equal to that of $f$ for any positive vector $f$. This, of course, follows from theorem 1 . To analyze the case of equality in (7), it suffices to assume $B$ is completely irreducible. In this event, it follows from theorem 1 and the fact that $B f$ has no 0 components that equality in (7) implies that all entries of $f$ are the same. Thus $D=F$ and equality holds in (7) precisely when $A$ is already doubly stochastic.

Note: A related but rather different inequality when $A$ is symmetric appears in [3, theorem 3$]$. Also a portion of the proof of that result could be used to prove part of the first statement in our theorem 1.

It follows from theorem 2 that

Conollary 2: If $\mathrm{A}$ is a row stochastic matrix with property $\left({ }^{*}\right)$ and $\mathrm{B}$ is related to $\mathrm{A}$ by $(1)$, then $|\operatorname{det} \mathrm{A}| \leq|\operatorname{det} \mathrm{B}|$. 
We denote the eigenvalues of $A$ by $a_{1}, \cdots, a_{n}$, ordered so that $\left|a_{1}\right| \leq \cdots \leq\left|a_{n}\right|$, and those of $B$ by $\beta_{1}, \cdots, \beta_{n}$, ordered so that $\left|\beta_{1}\right| \leq \cdots \leq\left|\beta_{n}\right|$. Since $a_{n}=1=\beta_{n}$, it follows from corollary 2 that

Corollary 3: If A is a row stochastic matrix with property $\left({ }^{*}\right)$ and $\mathrm{B}$ is related to $\mathrm{A}$ by $(1)$, then $\prod_{i=1}^{n-1}\left|a_{i}\right| \leq \prod_{i=1}^{n-1}\left|\beta_{i}\right|$.

We conjecture that in case $A$ is row stochastic with property $\left({ }^{*}\right)$ and $B$ is related to $A$ by (1), then actually

$$
\left|a_{i}\right| \leq\left|\beta_{i}\right|, i=1, \cdots, n \text {. }
$$

The result of theorem 2 may be extended to all matrices with property $\left({ }^{*}\right)$ in the following way.

Theorem 3: If $\mathrm{A}$ is any nonnegative $\mathrm{n}$ by $\mathrm{n}$ matrix with property $\left({ }^{*}\right)$ and $\mathrm{D}$ and $\mathrm{E}$ are positive diagonal matrices guaranteed by (1), then $\operatorname{det} \mathrm{DE} \geq \rho(\mathrm{A})^{-\mathrm{n}}$. Furthermore, equality holds only if $\mathrm{DE}=\rho(\mathrm{A})^{-1} \mathrm{I}$.

Proof: It is enough to assume $A$ is completely irreducible (for, if not, it is equivalen to a direct sum of same) and then $A$ is irreducible. In this event there is a positive vector $x$ such that $A x=\rho(A) x$ and, therefore, $\frac{1}{\rho(A)} X^{-1} A X$ is row stochastic, where $X=\operatorname{diag}\left\{x_{1}, \cdots, x_{n}\right\}$. Application of theorem 2 to $\frac{1}{\rho(A)} X^{-1} A X$ yields det $D^{\prime} E^{\prime} \geq 1$ where $D^{\prime}\left(\frac{1}{\rho(A)} X^{-1} A X\right) E^{\prime}=B$. Setting $D=\frac{1}{\rho(A)} D^{\prime} X^{-1}$ and $E=X E^{\prime}$, gives $B=D A E$ and det $D E \geq \rho(A)^{-n}$ as was to be shown. The case of equality also follows from theorem 2.

REMARK 3: The reader may wish to note the relationship between the present work and the notion of the equilibrant,

$$
E(B) \equiv \inf \rho(F B)
$$

(where the inf is taken over all positive diagonal matrices of determinant 1), of a nonnegative matrix mentioned in [5].

\section{References}

[1.] Brualdi, R., Parter, S., and Schneider, H., The diagonal equivalence of a nonnegative matrix to a stochastic matrix, J. Math. Anal. and Appl. 16, 31-50 (1966).

[2.] Hardy, G., Littlewood, J., Pólya, G., Inequalities, (Cambridge University Press, 1959).

[3.] Marcus, M., Newman, M., Generalized functions of symmetric matrices, Proc. AMS 16, 826-830, (1965).

[4.] Sinkhorn, R., A relationship between arbitrary positive matrices, and doubly stochastic matrices, Ann. Math. Stat. 35, 876-879, (1964).

[5.] Hoffman, A., Linear G-functions, Lin. and Multilin. Alg. 3, 45-52, (1975).

(Paper 80B4-454) 